\title{
BMJ Open Reducing time to differentiated service delivery for newly diagnosed people living with HIV in Kigali, Rwanda: study protocol for a pilot, unblinded, randomised controlled study
}

\author{
Jonathan Ross (D) , ${ }^{1,2}$ Gad Murenzi, ${ }^{3}$ Sarah Hill, ${ }^{2}$ Eric Remera (D) , \\ Charles Ingabire, ${ }^{3}$ Francine Umwiza, ${ }^{3}$ Athanase Munyaneza, ${ }^{3}$ Benjamin Muhoza, ${ }^{3}$ \\ Dominique Savio Habimana, ${ }^{4}$ Placidie Mugwaneza, ${ }^{4}$ Chenshu Zhang, ${ }^{1,2}$ \\ Marcel Yotebieng, ${ }^{1,2}$ Kathryn Anastos (D) ${ }^{1,2}$
}

To cite: Ross J, Murenzi G, Hill S, et al. Reducing time to differentiated service delivery for newly diagnosed people living with HIV in Kigali, Rwanda: study protocol for a pilot, unblinded, randomised controlled study. BMJ Open 2021;11:e047443. doi:10.1136/ bmjopen-2020-047443

- Prepublication history and additional supplemental material for this paper are available online. To view these files, please visit the journal online (http://dx.doi.org/10.1136/ bmjopen-2020-047443).

Received 30 November 2020 Revised 27 March 2021 Accepted 08 April 2021

Check for updates

(c) Author(s) (or their employer(s)) 2021. Re-use permitted under CC BY-NC. No commercial re-use. See rights and permissions. Published by BMJ.

For numbered affiliations see end of article.

Correspondence to

Dr Jonathan Ross;

joross@montefiore.org

\section{ABSTRACT}

Introduction Current HIV guidelines recommend differentiated service delivery (DSD) models that allow for fewer health centre visits for clinically stable people living with HIV (PLHIV). Newly diagnosed PLHIV may require more intensive care early in their treatment course, yet frequent appointments can be burdensome to patients and health systems. Determining the optimal parameters for defining clinical stability and transitioning to less frequent appointments could decrease patient burden and health system costs. The objectives of this pilot study are to explore the feasibility and acceptability of (1) reducing the time to DSD from 12 to 6 months after antiretroviral therapy (ART) initiation,and (2) reducing the number of suppressed viral loads required to enter DSD from two to one.

Methods and analyses The present study is a pilot, unblinded trial taking place in three health facilities in Kigali, Rwanda. Current Rwandan guidelines require PLHIV to be on ART for $\geq 12$ months with two consecutive suppressed viral loads in order to transition to less frequent appointments. We will randomise 90 participants to one of three arms: entry into DSD at 6 months after one suppressed viral load ( $n=30)$, entry into DSD at 6 months after two suppressed viral loads $(n=30)$ or current standard of care $(n=30)$. We will measure feasibility and acceptability of this intervention; clinical outcomes include viral suppression at 12 months (primary outcome) and appointment attendance (secondary outcome).

Ethics and dissemination This clinical trial was approved by the institutional review board of Albert Einstein College of Medicine and by the Rwanda National Ethics Committee. Findings will be disseminated through conferences and peer-reviewed publications, as well as meetings with stakeholders.

Trial registration number NCT04567693.

\section{INTRODUCTION}

With the goal of ending the HIV pandemic, the Joint United Nations Programme on HIV
Strengths and limitations of this study

- This pilot, randomised, controlled trial examining clinical outcomes of newly diagnosed people living with HIV who transition to differentiated service delivery (DSD) models after shorter intervals in care or fewer viral load measurements will provide important evidence to inform HIV programme implementation in Rwanda as well as globally.

- A three-armed study will be able to simultaneously explore the impact of (1) reducing the time to DSD from 12 to 6 months after antiretroviral therapy initiation and (2) reducing the number of suppressed viral load measurements required to enter DSD from two to one.

- Consideration of experienced and anticipated stigma as well as patient expenditures will provide additional information on the feasibility and acceptability of this model of care.

- The unblinded nature of this trial may lead to bias in subsequent clinical management and outcome ascertainment.

- The setting of the trial (urban health facilities in the capitol city of a country with a highly functional HIV care service delivery system and with a lower HIV prevalence than in much of Southern Africa) may limit the generalisability of our findings.

and AIDS (UNAIDS) '90-90-90' targets for 2020 are that $90 \%$ of all people living with HIV (PLHIV) know their HIV status; $90 \%$ of people with diagnosed HIV infection receive sustained antiretroviral therapy (ART); and $90 \%$ of all people receiving ART achieve viral suppression. ${ }^{1}$ To this end, in 2015, the WHO recommended in its Treat All guidelines that all PLHIV initiate ART as quickly as possible after diagnosis. ${ }^{2}$ Since implementation of its Treat All policy in 2016, Rwanda has nearly 
achieved UNAIDS 90-90-90 targets, ${ }^{3}$ yet groups including men and younger PLHIV remain at higher risk of poorer outcomes. Reducing barriers to initiating and adhering to therapy is thus paramount to ensuring all PLHIV in Rwanda succeed in HIV therapy.

The 2016 WHO guidelines recommend differentiated service delivery (DSD) models as a strategy to manage diverse sets of patient needs. ${ }^{2}$ Under these guidelines, PLHIV considered to be clinically stable-on ART for 1 year with two consecutive suppressed viral loads-can be seen less frequently for clinical assessments and dispensed ART for longer periods. Such approaches are feasible and acceptable, and achieve equivalent or improved retention in care and viral suppression. ${ }^{4-8}$ To date, numerous countries in sub-Saharan Africa have adopted DSD models, ${ }^{9}$ and some have modified eligibility for these programmes in response to the COVID-19 pandemic as a means to promote social distancing. ${ }^{10}$ While most DSD programmes limit eligibility to patients who are clinically stable, heterogeneity exists with respect to definitions of stability. Some programmes in sub-Saharan Africa use shorter intervals (ie, 6 months after ART initiation) and/ or only require a single suppressed viral load for categorisation as stable..$^{91-13}$ To date, most studies of DSD models have been limited to clinically stable patients, and no studies have empirically compared clinical outcomes of newly diagnosed PLHIV who transition to DSD models after shorter intervals in care or fewer viral load measurements compared with the current standard of care.

To optimise HIV programme outcomes under Treat All, Rwanda simultaneously introduced DSD models to align services with the variable needs and preferences of different groups of PLHIV. ${ }^{14}$ Stable PLHIV_adults on first-line or second-line ART for $\geq 12$ months with two consecutive suppressed viral loads-can collect ART every 3 months (rather than monthly) and attend clinical appointments every 3 or 6 months based on clinical criteria (table 1). Individuals in the unstable category-including newly diagnosed PLHIV ( $<12$ months on ART), women who are pregnant or lactating, patients with concurrent mental health disorders and PLHIV who are not virally suppressed-must visit the clinic monthly for ART collection and adherence assessment.

Our earlier research in Rwanda identified frequent appointments as burdensome to newly diagnosed PLHIV because of structural issues such as transportation cost and long wait times, as well as stigma experienced while travelling to and while at the health centre. ${ }^{15}$ Modifying the definition of clinically stable adults living with HIV to decrease the time on ART and reduce the number of viral load measurements could potentially reduce the burden faced by patients and health systems. However, implementing DSD earlier in patients' treatment may not provide them with the support needed to become stable in care and achieve viral suppression.

We are therefore conducting a pilot, unblinded, three-arm randomised controlled trial to explore the impact of two less intensive DSD models: (1) reducing the time to DSD from 12 to 6 months after ART initiation and (2) reducing the number of suppressed viral load measurements required to enter DSD from two to one. Our objectives were to understand whether these less-intensive DSD models are acceptable to participants and stakeholders, to determine whether their implementation is feasible in the context of current Rwandan HIV guidelines and to obtain parameter estimates to guide future efficacy testing. This study will contribute relevant information and actionable information to inform DSD care delivery in Rwanda and help plan for a future, fully powered study to test these models.

\section{METHODS AND ANALYSIS}

Trial design

This three-arm, unblinded, parallel group randomised controlled trial will examine the feasibility and

Table 1 Current differentiated care delivery model in Rwanda

\begin{tabular}{|c|c|c|c|}
\hline & Standard of care & Differentiated service delivery & \\
\hline & Unstable & Stable A & Stable B \\
\hline Patient & $\begin{array}{l}\text { Patients on ART for }<12 \\
\text { months. } \\
\text { Severe mental health disorder. } \\
\text { Pregnant or lactating. } \\
\text { On ART but not virally } \\
\text { suppressed. } \\
\text { - Patients on third-line ART. } \\
\text { Children }<2 \text { years old. }\end{array}$ & $\begin{array}{l}\text { Adults on first-line and } \\
\text { second-line ART with two } \\
\text { consecutive suppressed viral } \\
\text { loads. }\end{array}$ & $\begin{array}{l}\text { Children } \geq 2 \text { years. } \\
\text { Adolescents. } \\
\text { Key populations. } \\
\text { Coinfected with TB or } \\
\text { hepatitis. }\end{array}$ \\
\hline Provider & Clinical nurse & & \\
\hline Service location & Health centre & & \\
\hline Frequency of clinical visits & Every 3 months & Every 6 months & Every 3 months \\
\hline
\end{tabular}

ART, antiretroviral therapy; TB, tuberculosis. 
acceptability of reducing the time to DSD from 12 to 6 months as well as reducing from two to one the number of suppressed viral loads required to enter DSD, compared with usual care. The primary (viral suppression at 12 months after ART initiation) and secondary (appointment attendance over 12 months after ART initiation) efficacy outcomes will be compared using an exploratory, non-inferiority analysis.

\section{Study setting}

Rwanda, a landlocked nation with a population of nearly 13 million, became one of the first sub-Saharan African countries to implement Treat All nationally in 2016. The Rwandan HIV programme has been successful, with recent estimates of $>95 \%$ of PLHIV on ART and viral suppression of $>90 \% .{ }^{316}$ Rwanda has a pyramidal health system, with 8 national referral hospitals, 36 district hospitals and nearly 500 public health centres. Primary healthcare is predominantly delivered at health centres, which provide health promotion, preventive and treatment services in medicine, surgery, obstetrics and paediatrics, and are largely staffed by nurses. HIV care in Rwanda is decentralised and provided at nearly all health centres, and includes diagnostic testing, chronic disease management and ART. Current guidelines in Rwanda specify that all newly diagnosed patients should be on one of two ART regimens: tenofovir disoproxil fumarate, lamivudine and dolutegravir, or abacavir, lamivudine and dolutegravir. ${ }^{17}$

This study will be carried out in three health facilities located in Rwanda's capital city, Kigali: Gikondo Health Centre, Kicukiro Health Centre and Remera Health Centre. Together, these health facilities provide primary HIV care to approximately 6000 PLHIV, including approximately 300 newly diagnosed patients who enrol in care annually.

\section{Eligibility}

Inclusion criteria for this study are $(1) \geq 15$ years of age, (2) newly diagnosed with HIV within prior 6 months, (3) enrolled in HIV care at a participating study health facility within the prior 30 days and (4) initiated ART. Exclusion criteria are (1) planning on moving away from the Kigali area during the 12-month duration of study, (2) being pregnant or lactating at the time of study enrolment, (3) coinfection with active tuberculosis at the time of study enrolment, (4) concurrent known severe mental health or substance use disorder and (5) inability to provide informed consent.

\section{Interventions}

Participants will be randomised within 1 month of ART initiation to one of three arms in a 1:1:1 ratio, as follows:

Arm 1: entry into the DSD model at 6 months after ART initiation with one suppressed viral load. In this arm, participants will have their viral loads measured at 5 months after ART initiation. If the viral load is suppressed, they will advance to a spaced-out appointment schedule of clinical appointments every 6 months and ART pick-up every 3 months.

Arm 2: entry into the DSD model at 6 months after ART initiation with two suppressed viral loads. In this arm, participants will have viral loads measured at 3 and 5 months after ART initiation. If both are suppressed, they will advance to a spaced-out appointment schedule of clinical appointments every 6 months and ART pick up every 3 months. Because patients are expected to be on a dolutegravir-based regimen, we anticipate that those adherent to ART will have achieved viral suppression within 3 months of ART initiation.

Arm 3: usual care. In this arm, participants will have their viral loads measured at 5 months but will continue on an appointment schedule of clinical appointments every 3 months and ART pick-up monthly.

For participants in the intervention arms, the decision to advance patients to a DSD schedule is primarily contingent on their viral load measurements at 3 and/ or 5 months. However, healthcare providers at the health facilities may determine that patients are not eligible for a spaced-out appointment schedule based on overall clinical assessment and over-ride the study assignment. For example, individuals randomised to one of the intervention arms but who subsequently become ineligible for a DSD schedule because of pregnancy will not be permitted to continue in the DSD schedule and will cross to the usual care arm. Participants in the usual care arm will not be eligible for advancement to a DSD schedule until the study ends; however, those in the intervention arms may choose to attend appointments more frequently if agreed to by their clinician. All instances in which health centre staff determine that participants cannot advance to or continue in a spaced-out appointment schedule, including the reason for the change and whether it was initiated by the clinician or patient, will be recorded.

Before the study begins enrolment, staff at participating health facilities will undergo training on the study protocol, including eligibility criteria, study design and appointment schedules for the three arms. Throughout the study, the research team will regularly communicate with health facility staff to ensure that eligible study participants in the intervention arms advance to a spaced-out appointment schedule. The study team will review participant medical files to assess fidelity to the appointment schedule. While appointment schedules will be dictated by the study protocol, all other clinical treatments will be at the discretion of health facility clinicians. Following the trial, participants will continue in regular HIV care at their health facility; those in the usual care arm who were virally suppressed on preceding measurements will be eligible for advancement to a DSD schedule at this time.

\section{Outcomes}

To explore the impact of less frequent appointments and virological monitoring on patient outcomes, we will measure viral suppression (primary efficacy outcome) and appointment attendance (secondary efficacy 
outcome). Viral suppression will be measured as the proportion of participants in each arm who achieve viral suppression (viral load $<200$ copies $/ \mathrm{mL}$, based on current Rwandan guidelines) at 12 months after ART initiation. Appointment attendance will primarily be measured as the proportion of participants who attend all scheduled clinical and pharmacy visits over the first 12 months after ART initiation (11 in arms 1 and 2, 16 in arm 3) by reviewing participant medical records; we will also measure this outcome as the overall proportion of scheduled visits attended. Patients at study health centres who do not attend a scheduled appointment are called the next day to reschedule. If unsuccessful, appointments are considered missed; however, outreach efforts continue to be made.

Feasibility of an early spaced-out appointment schedule and less frequent virological monitoring will also be examined using process measures, including the proportion eligible, the proportion that provided consent, the proportion randomised, the proportion of participants attending appointments in each arm and cost measures. We will also conduct structured interviews with health facility staff at the end of the study to determine the feasibility of implementing this intervention at a larger scale.

Acceptability of an early spaced-out appointment schedule and less frequent virological monitoring will be measured through (1) surveys of satisfaction with healthcare, ${ }^{18} 19$ (2) structured qualitative interviews with patients and health facility staff in all arms to understand attitudes towards and satisfaction with various appointment schedules, (3) review of instances in which health centre clinicians over-ride the experimental assignment and (4) review of adverse event logs.

We will also measure within-group change over time and differences between groups for the following tertiary outcomes:

- ART adherence will be collected using self-reported ART adherence measures of 7 and 30 days at study entry, 6 and 12 months after ART initiation.

- Participant quality of life will be measured by the EuroQOL-5 Dimension-5 Levels, ${ }^{20}$ which measures self-rated problems in five domains (mobility, selfcare, usual activities, pain/discomfort and anxiety/ depression) as well as self-rated health. We will collect and report changes in quality of life at study entry, 6 and 12 months after ART initiation.

- Enacted, internalised and anticipated stigma will be measured using a modified version of the HIV Stigma Scale, ${ }^{19}$ as well as the HIV/AIDS Stigma InstrumentPLWA Scale. ${ }^{21}$ We will measure stigma at study entry, 6 and 12 months after ART initiation.

- Participant health-related expenditures will be measured at study entry, 6 and 12 months after ART initiation.
STUDY PROCEDURES

\section{Recruitment}

Active recruitment will occur via health facility nurses who will inform potentially eligible patients about the study during their routine appointments. Each week, a designated health facility staff member will provide the research assistant with a list of patients who indicated interest in participating and who meet the eligibility criteria (ie, newly diagnosed, not pregnant or lactating, without severe mental health conditions). Passive recruitment will occur through research assistants who will also make general announcements about the study during morning health education sessions at health facilities, and be available to answer questions and collect interested patient's contact information. Interested patients will be screened by study staff for eligibility and, if eligible, will be offered enrolment in the study.

\section{Study timeline}

The study enrolment visit will occur within 30 days of the patient's enrolment in HIV care at the health centre. All participants will have additional research visits 6 and 12 months after enrolling in HIV care. Research visits will entail participant interviews and medical record review. Participants will also visit the health facility for viral load measurements at 3 and 5 months after ART initiation, depending on the study arm. Participants will be reimbursed for all research and viral load visits. Table 2 describes the schedule of clinical and research visits. At the conclusion of the study enrolment visit, the research assistant will give the participant a reminder card with the date of the next research visit. Study staff will call the participant 1 week and 1 day before the scheduled research visits to remind them of the appointment date and time. Participants who do not appear for scheduled research visits will be called and visits will be rescheduled within 14 days. Research staff will not provide reminders for clinical or pharmacy visits.

\section{Informed consent}

Newly diagnosed PLHIV who have enrolled in care within 30 days and meet eligibility criteria will be referred to the study team. At study entry, written informed consent to participate will be obtained from all participants (online supplemental file 1, Model consent form). Participants aged 15-18 years will provide assent with informed consent obtained from their parent or legal guardian. Research staff will read the informed consent document to participants in its entirety; participants unable to sign their name will be permitted to sign with an 'X.' No additional consent provisions are required for collection and use of participant medical record data and biological specimens in this study.

\section{Randomisation}

At study entry, participants will be randomised to one of three study arms. To ensure equal distribution of key factors among randomisation arms, we will stratify 
Table 2 Schedule of health centre and research visits

\section{Health centre and research visits after ART initiation (months)}

\begin{tabular}{|c|c|c|c|c|c|c|c|c|c|c|c|c|}
\hline Timepoint (month) & 1 & 2 & 3 & 4 & 5 & 6 & 7 & 8 & 9 & 10 & 11 & 12 \\
\hline \multicolumn{13}{|c|}{ Intervention: appointment and viral load schedule } \\
\hline \multicolumn{13}{|c|}{ Arm 1 (early DSD after one suppressed viral load) } \\
\hline ART pick-up & $\bullet$ & $\bullet$ & $\bullet$ & $\bullet$ & $\bullet$ & • & & & $\bullet$ & & & $\bullet$ \\
\hline Viral load measurement & & & & & $\bullet$ & & & & & & & - \\
\hline Clinical appointments & & & $\bullet$ & & & $\bullet$ & & & & & & $\bullet$ \\
\hline ART pick-up & $\bullet$ & $\bullet$ & $\bullet$ & $\bullet$ & $\bullet$ & $\bullet$ & & & $\bullet$ & & & $\bullet$ \\
\hline Viral load measurement & & & $\bullet$ & & $\bullet$ & & & & & & & $\bullet$ \\
\hline \multicolumn{13}{|l|}{ Arm 3 (usual care) } \\
\hline Clinical appointments & & & & & & $\bullet$ & & & $\bullet$ & & & $\bullet$ \\
\hline Research visits (all arms) & $\bullet$ & & & & & $\bullet$ & & & & & & $\bullet$ \\
\hline
\end{tabular}

$\mathrm{ART}$, antiretroviral therapy; DSD, differentiated service delivery.

randomisation by age group (younger or older than 24 years) and health facility. We will randomise in blocks to ensure comparison groups of approximately equal size. Randomisation will be computer generated, occur in blocks of six with 1:1:1 allocation across study arms.

To ensure concealment of allocation, a centrally located data manager will generate the allocation sequence and store the sequence in a password-protected file. Since the intervention is not blinded, we will use block size of 6 to prevent anticipation of treatment arm assignment. The allocation sequence will be generated using the Proc Plan function in SAS V.9.4. On enrolment in the study, research staff will use the randomisation function in Research Electronic Data Capture (REDCap) V.10.0.16, 2020 (Vanderbilt University), to assign participants to study arms. Because this study is examining the effect of different appointment schedules, it is not feasible to blind participants or study personnel, and thus allocation will not be concealed from staff or participants.

\section{Data collection}

Data will be collected through participant interviews, laboratory tests and medical record review. Research staff will be trained in systematic data collection by interview. Interviews will be conducted in Kinyarwanda by staff with responses entered directly into REDCap. Downtime protocols will be implemented in the event of internet outage. Medical records will be reviewed at the end of every study visit, with data entered directly into REDCap. Venous blood specimens for clinical monitoring (eg, CD4 count and viral load) will be collected at study entry and at several subsequent visits during the study. Results will be provided to clinical staff at health facilities, who will input them into the medical record and report them to participants, consistent with routine clinical practices. Viral load measurements will be performed using the Abbott Allinity m instrument, with a lower limit of detection of 20 copies/mL. No genetic or molecular analyses will be performed; specimens will not be stored for future use.

\section{Analytical approach}

We expect to enrol 90 participants into this study. This pilot study is designed to test feasibility and acceptability and is thus not powered for hypothesis testing. Sample size was determined based on available resources for conducting the study. The primary analyses will be by intention to treat.

Data obtained through REDCap will be imported into SAS V.9.4. We will first clean the data, examining frequencies, means, medians and ranges to identify any systematic or logical errors. As this is a pilot study, analyses will be descriptive in nature. Validated instruments will be coded according to respective scoring instructions. Feasibility will be examined using descriptive analyses to describe process measures including the proportion eligible, the proportion that provided consent, the proportion randomised, the proportion of participants attending appointments in each arm and cost measurements. We will also conduct thematic analysis of qualitative, structured interviews to determine intervention acceptability of less intensive DSD models as well as feasibility of implementing this intervention at a larger scale.

For the outcomes of viral suppression and appointment adherence, the primary analysis will be intention to treat, including all randomised participants, with those who are missing outcome data considered treatment failures. We will first compare study arms with respect to the 
proportions of patients achieving viral suppression and attending all clinical and pharmacy visits using $\chi^{2}$ tests. We will then use logistic regression to estimate ORs and associated 95\% CIs for the effect of each intervention arm compared with the control, adjusting for key baseline covariates that are imbalanced between groups. Because of the small number of participants we anticipate enrolling in this pilot study, we will not be sufficiently powered to detect statistically significant differences in outcomes. However, the findings obtained from this study will provide key results on intervention feasibility and guide a future, larger study to test intervention efficacy.

Due to the pilot nature of this study, relatively short duration and small planned enrolment size, we do not plan on conducting interim analyses. In secondary analyses, we will examine outcomes of viral suppression and appointment attendance using a per-protocol approach. We will also compare statistical results using a dataset with imputed values and the dataset that drops missing values, guiding our interpretation of the impact of missing data on findings, as well as our interpretation of overall results. Moreover, because outcome data may be missing for different reasons, we will document reasons for missingness to the degree possible to inform these additional analyses. For example, while the number of deaths is anticipated to be small, we will conduct analyses both including and excluding participants who died to understand the potential impact of death on results. Additional subanalyses will examine outcomes separately among subgroups of interest (ie, men, young patients and early defaulters), though these may be limited by the small size of some subgroups.

\section{Data management}

In accordance with Rwandan research regulations, all personally identifying information, including participant names and contact information, will be collected using a locally stored, password-protected, encrypted database. REDCap will be used to securely collect, validate (eg, range checks and logical dates) and store interview, medical record and laboratory data. No identifying information will be collected in REDCap. Only research investigators and staff will have access to study databases. Data quality will be promoted through training research staff to uniformly collect and enter data and by periodic data quality monitoring.

\section{Confidentiality}

The following measures will be used to protect participant confidentiality: all paper study records (ie, informed consent documents) will be kept in locked file cabinets with access limited to study staff. In accordance with Rwandan research regulations, all personally identifying information, including participant names and contact information, will be collected using a locally stored, password-protected, encrypted database. REDCap data will not include any name-based or identifying information. Study databases will be maintained on encrypted, password-protected computers and servers to which only study staff will have access. To prevent linking of sensitive material to participants' personal identifiers, we will use separate 'name-based' and 'ID-based' systems. For any paper forms, all documents that have patient identifiers (eg, consent forms and locator forms) will be filed together. Any files that do not include identifying information or signatures will only use participants' unique, study-specific IDs (rather than names) and will be filed separately from name-based documents. There will only be one electronic document that links participants' names to their study IDs, stored on a local, passwordprotected, encrypted server. Publication or presentation of study results will not identify subjects.

\section{Study oversight}

This is a pilot study of approximately 90 participants being conducted to test feasibility and acceptability of a modified appointment schedule. This is a low-risk study that involves pilot testing an intervention that will enrol a relatively small number of participants, and it is unlikely that study participants will experience adverse reactions related to study participation. Therefore, there will be no data safety and monitoring board. The study team, consisting of the principal investigator (PI), coinvestigators and research staff, will meet weekly to review study progress, including review of adverse events. If adverse events occur, the PI will act to minimise their impact and ensure the adverse event is reported to the responsible authorities in a timely manner as required. There is no coordinating centre or steering committee. This pilot trial will not be audited.

\section{Adverse event reporting and harms}

The PI, together with the study team, will be responsible for regularly monitoring data and safety, specifically assessing for adverse events and breach of confidentiality. If adverse events occur, the study team will (1) identify the concern, (2) activate the appropriate response to minimise the adverse event and (3) ensure the adverse event is reported to the responsible authority in a timely manner. If patients have a medical or psychiatric decompensation during the study, research staff will inform their direct supervisor, who will assess the patient in person and will notify the PI or coinvestigators immediately. Based on clinical judgement, study participants will be referred to psychiatric or medical consultation in the health facility or referred for emergency care. The study database will be secured with encryption and password protection, and the study team will monitor the database for potential breaches of confidentiality.

All adverse events will be compiled monthly. Unanticipated, non-serious adverse events will be documented and reported by the PI to the Albert Einstein College of Medicine institutional review board (IRB) and the Rwanda National Ethics Committee within 30 days. Serious adverse events will be reported by the PI to the Albert 
Einstein College of Medicine IRB and Rwanda National Ethics Committee within 48 hours by phone, email or fax.

\section{DISCUSSION}

This trial will pilot test reducing the time to DSD from 12 to 6 months as well as reducing the number of suppressed viral loads required to enter DSD from two to one, compared with usual care. If found feasible and acceptable, this approach could reduce inconvenience and stigma for newly diagnosed PLHIV as well as lower the medical resources required for treatment.

This study may face potential limitations. Participants in the intervention arm with two viral load measurements effectively have twice as many opportunities to not be virally suppressed, with viral suppression less likely at 3 months compared with 5 months, and therefore may be less likely to advance to DSD. Similarly, participants will advance to DSD at the discretion of treating clinicians. This may bias the study findings if participants in the intervention arms effectively follow the standard of care and may result in less power to detect differences between the study arms in the intention to treat analysis. Nonetheless, the study will provide important feasibility and acceptability data on the optimal number of viral load measurements needed to determine clinical stability. Because inclusion criteria specify HIV diagnosis within the preceding 6 months, it is possible that participants will have been in care elsewhere and defaulted prior to enrolling in study health centres, and thus are not ART-naive. While it may not be possible to know if patients are truly newly diagnosed, we expect that any early defaulters would be equally distributed between arms, given the randomised nature of this study, and thus would not impact analysis of outcomes. Blinding will not be feasible for this study, which may bias the study findings. ART adherence will be measured by self-report, which may imperfectly reflect true medication adherence. Finally, we will be enrolling patients who receive care at health facilities located in or near the capital of a country with a highly functional HIV care service delivery system and with a lower HIV prevalence than in much of Southern Africa. This may limit the generalisability of our findings.

DSD models, including less frequent appointment schedules, are increasingly being adopted across HIV care settings globally, and are acceptable to patients and cost-effective. ${ }^{4-82223}$ A key question in implementing DSD models is determining at what point patients receiving HIV care can be considered clinically stable. By examining the effect of reduced time to DSD as well as fewer viral load measurements prior to entering DSD, this study will provide key parameters for a subsequent, larger efficacy trial, and will provide practical data for HIV programme implementation in Rwanda as well as globally.

\section{Patient and public involvement}

The design of this study was informed by findings of formative, qualitative research conducted with patients at study health centres, ${ }^{15}$ clinical experiences of several of the authors, along with input from public health and clinical leaders in Rwanda. The research questions and design were reviewed by investigators with expertise in HIV health service delivery and infectious diseases, along with input from an advisory committee consisting of leadership from study health centres as well as the Rwanda Biomedical Centre, the nation's central health implementation agency.

\section{ETHICS AND DISSEMINATION}

This clinical trial was approved by the IRB of Albert Einstein College of Medicine and by the Rwanda National Ethics Committee and is registered at www.clinicaltrials. gov.

By examining the effect of reduced time to DSD as well as fewer viral load measurements prior to entering DSD, this study will provide key parameters for a subsequent, larger efficacy trial, and will provide practical data for HIV programme implementation in Rwanda as well as globally.

We will disseminate study findings through presentations at scientific conferences, publications in peerreviewed journals, and presentations to patients, providers and key institutional stakeholders. Study findings will be reported in accordance with the Consolidated Standards of Reporting Trials.

\section{TRIAL STATUS}

The current protocol is V.1.4, dated 28 September 2020. Any important protocol amendments will be communicated immediately to the responsible ethical committees and will be reported in resulting publications. Recruitment for this trial began on 22 October 2020 and is expected to continue until August 2021, with follow-up continuing until August 2022.

\section{Author affiliations}

${ }^{1}$ Division of General Internal Medicine, Montefiore Health System, Bronx, New York, USA

${ }^{2}$ Division of General Internal Medicine, Yeshiva University Albert Einstein College of Medicine, Bronx, New York, USA

${ }^{3}$ Clinical Education and Research Division, Rwanda Military Hospital, Kigali, Kigali City, Rwanda

${ }^{4}$ Institute of HIV Disease Prevention and Control, Rwanda Biomedical Center, Kigali, Rwanda

Contributors $\mathrm{JR}$ is the principal investigator, conceived the study, and led the proposal and protocol development. SH, CI, FU, AM, BM, GM and KA contributed to the study design and to development of the proposal. ER, DSH, PM and MY provided additional input to the study design. CZ provided statistical support. All authors read and approved the final manuscript.

Funding This work was supported by the US National Institute of Mental Health (K23 MH114752); by the US National Institutes of Health's (NIH) National Institute of Allergy and Infectious Diseases, the Eunice Kennedy Shriver National Institute of Child Health and Human Development, the National Cancer Institute, the National Institute of Mental Health and the National Institute on Drug Abuse, as part of Central Africa leDEA (U01 Al096299); and by the Einstein-Rockefeller-CUNY Center for AIDS Research (P30 Al124414), which is supported by the following $\mathrm{NIH}$ cofunding and participating institutes and centres: National Institute of 
Allergy and Infectious Diseases, National Cancer Institute, Eunice Kennedy Shriver National Institute of Child Health and Human Development, National Heart, Lung and Blood Institute, National Institute on Drug Abuse, National Institute of Mental Health, National Institute on Aging, Fogarty International Center, and Office of AIDS Research. The content is solely the responsibility of the authors and does not necessarily represent the official views of the NIH. The study is sponsored by Montefiore Medical Center (111 E. 210th St., Bronx, NY 10467, +1.718.920.4321). The study sponsor and funder have no role in the study design; collection, management, analysis and interpretation of data; writing of the report; and the decision to submit the report for publication.

Competing interests None declared.

Patient consent for publication Not required.

Provenance and peer review Not commissioned; externally peer reviewed.

Supplemental material This content has been supplied by the author(s). It has not been vetted by BMJ Publishing Group Limited (BMJ) and may not have been peer-reviewed. Any opinions or recommendations discussed are solely those of the author(s) and are not endorsed by BMJ. BMJ disclaims all liability and responsibility arising from any reliance placed on the content. Where the content includes any translated material, BMJ does not warrant the accuracy and reliability of the translations (including but not limited to local regulations, clinical guidelines, terminology, drug names and drug dosages), and is not responsible for any error and/or omissions arising from translation and adaptation or otherwise.

Open access This is an open access article distributed in accordance with the Creative Commons Attribution Non Commercial (CC BY-NC 4.0) license, which permits others to distribute, remix, adapt, build upon this work non-commercially, and license their derivative works on different terms, provided the original work is properly cited, appropriate credit is given, any changes made indicated, and the use is non-commercial. See: http://creativecommons.org/licenses/by-nc/4.0/.

ORCID iDs

Jonathan Ross http://orcid.org/0000-0001-6126-3048

Eric Remera http://orcid.org/0000-0002-6084-8877

Kathryn Anastos http://orcid.org/0000-0002-0769-6405

\section{REFERENCES}

1 Joint United Nations Programme on HIV/AIDS. 90-90-90: an ambitious treatment target to help end the AIDS epidemic. Geneva, Switzerland, 2014.

2 World Health Organization, Department of HIV/AIDS. Consolidated guidelines on the use of antiretroviral drugs for treating and preventing HIV infection 2016: recommendations for a public health approach. 2nd edn. Geneva, Switzerland: World Health Organization, 2016.

3 Rwanda summary sheet, population-based HIV health impact assessment [online]. Available: https://phia.icap.columbia.edu/ rwanda-summary-sheet/ [Accessed 6 Oct 2020].

4 Phiri K, McBride K, Siwale Z, et al. Provider experiences with threeand six-month antiretroviral therapy dispensing for stable clients in Zambia. AIDS Care 2021;33:1-7.

5 Eshun-Wilson I, Mukumbwa-Mwenechanya M, Kim H-Y, et al. Differentiated care preferences of stable patients on antiretroviral therapy in Zambia: a discrete choice experiment. J Acquir Immune Defic Syndr 2019;81:540-6.

6 Long L, Kuchukhidze S, Pascoe S, et al. Retention in care and viral suppression in differentiated service delivery models for HIV treatment delivery in sub-Saharan Africa: a rapid systematic review. $J$ Int AIDS Soc 2020;23:e25640.

7 Fatti G, Ngorima-Mabhena N, Mothibi E, et al. Outcomes of three- versus sexi-monthly dispensing of antiretroviral treatment (ART) for stable HIV patients in community ART refill groups: a cluster-randomized trial in Zimbabwe. J Acquir Immune Defic Syndr 2020;84:162-72.

8 Tukei BB, Fatti G, Tiam A, et al. Twelve-month outcomes of community-based differentiated models of multimonth dispensing of ART among stable HIV-infected adults in lesotho: a clusterrandomized noniferiority trial. J Acquir Immune Defic Syndr 2020;85:280-91.

9 Differentiated service delivery. Available: https://differentiatedservic edelivery.org [Accessed 5 Mar 2021].

10 U.S. Department of State. PEPFAR technical guidance in context of COVID-19 pandemic, 24 February 2021. Available: https://www. state.gov/wp-content/uploads/2021/02/02.24.21-PEPFAR-TechnicalGuidance-During-COVID.pdf [Accessed 5 Mar 2021].

11 Prust ML, Banda CK, Nyirenda R, et al. Multi-month prescriptions, fast-track refills, and community ART groups: results from a process evaluation in Malawi on using differentiated models of care to achieve national HIV treatment goals. J Int AIDS Soc 2017;20:21650.

12 Wringe A, Cawley C, Szumilin E, et al. Retention in care among clinically stable antiretroviral therapy patients following a six-monthly clinical consultation schedule: findings from a cohort study in rural Malawi. J Int AIDS Soc 2018;21:e25207.

13 PEPFAR Burundi country operational plan (COP) 2017 strategic direction summary April 29, 2017. Available: https://copsdata.amfar. org/SDS/2017/Burundi.pdf [Accessed 23 Oct 2020].

14 Rwanda Biomedical Center. National guidelines for prevention and management of HIV and STIs. Kigali, Rwanda, 2016.

15 Ingabire C, Umwiza F, Gasana J. "It's a big problem to take that pill before you feel ready": ART initiation challenges under treat all in rwanda. oral presentation, international conference on AIDS and STDs in Africa. Kigali, Rwanda, 2019.

16 Ross J, Ribakare M, Remera E, et al. High levels of viral load monitoring and viral suppression under Treat All in Rwanda - a crosssectional study. J Int AIDS Soc 2020;23:e25543.

17 Rwanda Biomedical Center. Rwanda HIV and AIDS national strategic plan 2013-2018; extension 2018-2020. Rwanda: Kigail.

18 Lannes L. Improving health worker performance: the patientperspective from a PBF program in Rwanda. Soc Sci Med 2015;138:1-11.

19 Parcesepe A, Tymejczyk O, Remien R, et al. HIV-related stigma, social support, and psychological distress among individuals initiating art in Ethiopia. AIDS Behav 2018;22:3815-25.

20 van Hout B, Janssen MF, Feng Y-S, et al. Interim scoring for the EQ$5 \mathrm{D}-5 \mathrm{~L}$ : mapping the EQ-5D-5L to EQ-5D-3L value sets. Value Health 2012;15:708-15.

21 Holzemer WL, Uys LR, Chirwa ML, et al. Validation of the HIV/AIDS stigma instrument - PLWA (HASI-P). AIDS Care 2007;19:1002-12.

22 Mesic A, Fontaine J, Aye T, et al. Implications of differentiated care for successful ART scale-up in a concentrated HIV epidemic in Yangon, Myanmar. J Int AIDS Soc 2017;20:21644.

23 Roberts DA, Tan N, Limaye N, et al. Cost of differentiated HIV antiretroviral therapy delivery strategies in sub-Saharan Africa: a systematic review. J Acquir Immune Defic Syndr 2019;82:S339-47. 